\title{
Longitudinal study on the prevalence of benzodiazepine (mis)use in a prison: importance of the analytical strategy
}

\author{
D. Borrey', E. Meyer², L. Duchateau², W. Lambert', C. Van Peteghem' \& A. P. De Leenheer'* \\ Laboratorium voor Toxicologie, Universiteit Gent, Gent, Belgium' and Laboratorium voor Fysiologie, Biochemie en Biometrie, Universiteit Gent, Merelbeke, \\ Belgium²
}

Correspondence to:

Prof Dr A. P. De Leenheer

Laboratorium voor Toxicologie

Universiteit Gent

Harelbekestraat 72

B-9000 Gent

Belgium

Tel: 3292648121

Fax: 3292648183

E-mail: Andre.DeLeenheer@UGent.be

Submitted 8 January 2003;

initial review completed 20 May 2003:

final version accepted 26 June 2003

\begin{abstract}
Aims This study evaluates the suitability of gas chromatographic-mass spectrometric (GC-MS) analysis to follow-up the extent of benzodiazepine (mis)use in a Belgian prison population and compares it to other analytical strategies (e.g. screening followed by confirmation of the positive samples).

Design and participants From February to August 1998, 598 persons were jailed of which 188 (31.4\% of the incoming detainees) volunteered to be screened. Urine samples (530 in total) were collected on the day of arrival and after 14, 30 and 90 days of imprisonment.

Measurements All samples were screened by EMIT $^{\circledR}$ for benzodiazepines and analysed subsequently by GC-MS.

Findings EMIT $^{\circledR}$ screening yielded 117 (22.1\%) positive samples, a number which increased to 174 (32.8\%) after GC-MS analysis. Of these 174 GC-MS positive samples, 119 (68.4\%) contained one benzodiazepine while for the remaining samples multiple benzodiazepine (mis)use could be demonstrated. A significant increase in benzodiazepine (mis)use was indicated only from day 0 to day 14 based on the GC-MS results but not on the immunoassay results, even when the latter were complemented with GC-MS analysis of the positively screened samples. The GC-MS data also demonstrated that benzodiazepines are mainly (mis)used by subjects on benzodiazepine prescription as almost 50\% of these subjects took additional non-prescribed benzodiazepines. During GC-MS analysis other drugs were co-extracted unintentionally and chromatographed and $23.9 \%$ of the volunteers were positive for illegal drugs on the day of arrival. Conclusion Immunoassay results yield an underestimation of the problem of benzodiazepine (mis)use in prison due to the high false negative rate. GC-MS analysis of all samples therefore is the recommended strategy for this type of longitudinal study as it yields more correct and detailed information than the immunoassay results.
\end{abstract}

KEYWORDS Benzodiazepines, offender population, urine drug testing.

\section{INTRODUCTION}

In several European countries, drug users constitute a significant proportion of the prison population. The results of several studies indicate that the problem exists before detention and cannot be stopped by the prison walls $[1,2]$. Control of drugs entering the prison where this study was performed was carried out by checking the luggage of visitors and also by searching the prisoners before they returned to their cells. However, addicted prisoners use great ingenuity to smuggle drugs into prison and the measures taken are not always effective. In prison, drugs are circulating in subcultures and those who refuse to cooperate can fall victim of fights, 
aggression or threats. This atmosphere forces prisoners to use drugs and hampers the management of the institution. To be in control of the situation hypnotics and sedatives such as benzodiazepines are often prescribed and their use is widespread among prisoners. Both illegal and medical drug use can occur in prison [2-5].

Self-reported drug use is often under-reported when compared with the analysis of urine or hair [6-8]. Fraser et al. [9] described the results of the Canadian urine drug screening programme for 1999 and concluded that urine drug testing provided an objective measure of drug use by offenders in Canadian federal institutions and those living in the community on conditional release.

The capability of immunological screening and gas chromatographic-mass spectrometric (GC-MS) analysis of urine to evaluate the benzodiazepine (mis)use in a prison population was compared in a longitudinal study.

\section{MATERIALS AND METHODS}

\section{Samples}

Urine samples were collected in a Belgian house of detention for male prisoners between February and August 1998. Every imprisoned person was informed about the study and was asked to cooperate. An important task of the medical staff was to motivate people, although participation had to be on a voluntary basis. Participation in the study did not affect the prisoner treatments and written informed consent was obtained in each case. Urine samples were collected in accordance to the standards of the ethical review committee of the Ghent University Hospital. The prisoners to be tested were collected from their cells by a guard and brought to the physicians' room. Surveillance of a nurse was recommended to prevent dilution or adulteration of the samples. A total of 530 urine specimens were collected on four test days: on the day of arrival (day 0) and after 14 days (day 14), 1 month (day 30) and 3 months (day 90) of incarceration. The plastic containers were labelled with the serial number of the volunteer and the sampling day, and stored at $-20^{\circ} \mathrm{C}$ until analysis.

\section{METHODS}

All samples were tested for creatinine and screened for the presence of benzodiazepines by EMIT $^{\circledR}$. The kits were obtained from Syva Co. (Dade Behring Inc., Cupertino, CA, USA) and were used on a Cobas Mira Instrument (Roche, Basel, Switzerland). The detection limit (cut-off value) of the benzodiazepine assay corresponds to $0.2 \mathrm{mg} / \mathrm{l}$ of oxazepam, as specified in Syva's instructions.
The assay was calibrated daily with the low $(0.2 \mathrm{mg} / \mathrm{l}$ oxazepam) and high (1.0 mg/l oxazepam) calibrators and the calibration was validated by assaying positive and negative controls. Unknowns were determined from the calibration curve by linear interpolation. A sample that gave a change in absorbance $(\Delta \mathrm{A})$ equal to or higher than the low calibrator was interpreted as positive, a sample that gave a change in absorption higher than the high calibrator was diluted until the value was within the linear range.

Enzymatic hydrolysis was performed as an initial step for the subsequent solid-phase extraction and GC-MS analysis. The method was validated for oxazepam, a common metabolite of several benzodiazepines, and for 14 other benzodiazepine compounds [10,11]. As in many samples bromazepam, diazepam, prazepam and temazepam were also identified, the recoveries for these compounds were determined at three different concentrations: $5 \mathrm{ng} / \mathrm{ml}, 100 \mathrm{ng} / \mathrm{ml}$ and $1000 \mathrm{ng} / \mathrm{ml}(n=4)$. They ranged from $85 \%$ to $93 \%(\mathrm{CV} \%<12 \%)$, from $81 \%$ to $96 \%(\mathrm{CV} \%<11 \%)$, from $82 \%$ to $93 \%(\mathrm{CV} \%<14 \%)$ and from $86 \%$ to $95 \%(C V \%<10 \%)$, respectively, indicating that the optimized GC-MS procedure also allows reliable detection of these benzodiazepines. Interpretation of the GC-MS results was based on the limit of detection (LOD) for the studied benzodiazepines $(13-30 \mathrm{ng} / \mathrm{ml}$ ) while for all other drug compounds the spectra had to be in accordance with those from the used MS library for at least $85 \%$. Samples were interpreted negative for a specific (illegal) drug, if the spectral quality was lower.

\section{Statistical analysis}

Results of the benzodiazepine testing on day 14 was compared with day 0, day 30 with day 14 and day 90 with day 30 .

Immunological screening results in binary data (presence or absence of benzodiazepines) and analysis was based on McNemar's test [12]. The full GC-MS data can be summarized as the number of different benzodiazepines found and the analysis was based on the change in number of benzodiazepines taken using the signed rank test [13]. The effect of the false negative rate of the immunoassay compared to GC-MS was assessed by an additional analysis with dichotomized GC-MS results (GC-MS binary) using McNemar's test.

A strategy that is often used for screening consists of first determining the presence of benzodiazepines by an immunoassay, whereupon all positive samples are analysed further by GC-MS (EMIT/GC-MS). In order to compare this strategy with the more time-consuming strategy of determining all samples by GC-MS, an additional analysis of the EMIT/GC-MS data was performed based on the signed rank test with, however, all immunoassay negative 
samples scored as 0 for the number of benzodiazepines found.

\section{RESULTS}

During the test period a total of 598 people were jailed, of whom 188 (31.4\%) volunteered for the project. Decreasing numbers of samples were collected on follow-up test days due to remand or transfer of the prisoners (Table 1). Only one prisoner refused to cooperate further after 1 month of incarceration.

All samples were tested first for creatinine and nine (1.7\%) of them contained less than $20 \mathrm{mg} / \mathrm{dl}$ of creatinine (two specimens were collected on day 0 , three on day 14 , three on day 30 and one on day 90). From these dilute samples 5-ml instead of 1-ml aliquots were extracted prior to GC-MS analysis. No signs of adulteration (turbidity, foaming, altered $\mathrm{pH}$ ) [14] were detected.

The EMIT and GC-MS results of the benzodiazepine analyses are summarized in Table 1. With EMIT screening a total of 117 positive benzodiazepine tests (22.1\%) were obtained. All EMIT-positive samples also tested positive with GC-MS. For GC-MS, a greater rate of positivity (in total 174 or $32.8 \%$ ) was found. Statistical evaluation of these data is presented in Fig. 1 (more especially the statistical difference between day 14 versus day 0). The most frequently found benzodiazepines with GC-MS were oxazepam (14.0\%) and bromazepam (9.1\%). Lormetazepam (8.3\%), temazepam $(6.4 \%)$, diazepam $(4.5 \%)$ and alprazolam $(4.5 \%)$ were also commonly identified. Flurazepam (1.5\%), flunitrazepam (0.4\%) and prazepam $(0.4 \%)$ were detected in only a few samples.

The number of different benzodiazepines identified by GC-MS (primary and secondary agents) in the corresponding number of samples is listed in Table 2. From the 174 GC-MS-positive urine specimens, 119 (68.4\%) contained only one benzodiazepine, whereas in the others multiple benzodiazepine (mis)use was demonstrated. In one sample, up to seven different benzodiazepines could even be identified.

No significant change in benzodiazepine (mis)use from day 0 to day 14 could be demonstrated when basing McNemar's test on the EMIT results $(P=0.052)$ compared to a significant increase for the binary GC-MS results $(P<0.0001)$ (Fig. 1). Based on the analysis of the EMIT/GC-MS results or GC-MS full data, the change in number of benzodiazepines taken was evaluated using the signed rank test. Analysis of the EMIT/GC-MS results yielded no significant change in benzodiazepine (mis)use while with the GC-MS full data a significant increase in the number of benzodiazepines taken from day 0 to day $14(P=0.0003)$ was obtained, with 28 subjects taking one additional benzodiazepine $(+1)$ and 11 subjects taking more than one additional benzodiazepine $(+2$ or +3$)$. No significant differences were found for any of the tests

Table I Number of samples tested positive for benzodiazepines with EMIT or GC-MS.

\begin{tabular}{lccccc}
\hline & Day 0 & Day 14 & Day 30 & Day 90 & Total \\
\hline No. of samples & 188 & 170 & 135 & 37 & 530 \\
EMIT+ & $36(19.1 \%)$ & $40(23.5 \%)$ & $31(23.0 \%)$ & $10(27 \%)$ & $117(22.1 \%)$ \\
GC-MS+ & $49(26.1 \%)$ & $64(37.6 \%)$ & $49(36.3 \%)$ & $12(32.4 \%)$ & $174(32.8 \%)$ \\
\hline
\end{tabular}

Figure I Change in benzodiazepine use from day 0 to day 14 based on EMIT and GC-MS results. GC-MS binary: GC-MS data reduced to binary results. With these binary data it can only be investigated whether prisoners start (+I) or stop (-I) using benzodiazepines. EMIT-GCMS: EMIT screening with GC-MS confirmation of the positive screening results. Based on these data and the GC-MS results the difference in number of benzodiazepines found $(-2$ to +3 ) can additionally be examined (frequency equals the number of samples).

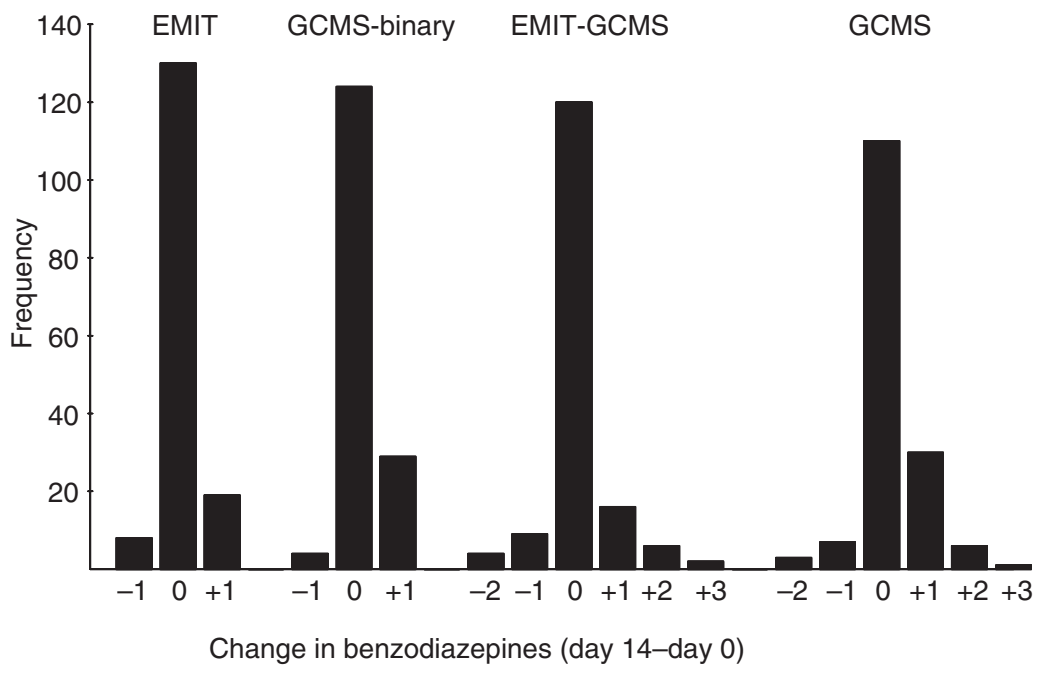


performed when comparing the (mis)use on day 30 versus day 14, and day 90 versus day 30 .

The EMIT- and GC-MS-positive results were also compared to the doctor's prescriptions to discriminate between legal and illegal benzodiazepine use. Samples collected on the day of arrival could not be used for this purpose as these data reflect only the benzodiazepine (mis)use of the volunteers before detention, and it was unknown whether or not the benzodiazepines found at that time were prescribed. Based on the EMIT results, 22 of 221 samples (10.0\%) were found positive, although no benzodiazepines were prescribed. For GC-MS, the number of positive samples was 41 (18.6\%). From the 103

Table 2 Number of different benzodiazepines identified in the samples.

\begin{tabular}{lccccc}
\hline \multicolumn{7}{l}{ No. of samples } \\
No. of & \multicolumn{7}{l}{} & & \\
\cline { 2 - 6 } benzodiazepines & Day 0 & Day 14 & Day 30 & Day 90 & Total \\
\hline I & 32 & 46 & 33 & 8 & 119 \\
2 & 12 & 12 & 7 & 3 & 34 \\
3 & 4 & 3 & 4 & 1 & 12 \\
4 & 1 & 3 & 4 & - & 8 \\
5 & - & - & - & - & - \\
6 & - & - & - & - & - \\
7 & - & - & 1 & - & 1 \\
Total & 49 & 64 & 49 & 12 & 174 \\
\hline
\end{tabular}

samples collected from prisoners who were prescribed benzodiazepines, $47(45.6 \%)$ contained no benzodiazepines by EMIT, 25 (24.3\%) by GC-MS. Remarkably, nonprescribed benzodiazepines were detected in 50 (48.5\%) of 103 samples from subjects who were prescribed a particular benzodiazepine. A detailed comparison between the GC-MS full data and the benzodiazepines prescribed is summarized in Table 3.

Finally, the frequency of positive findings for other legal and illegal drug classes by GC-MS analysis is found in Table 4. These compounds were not searched for intentionally and prescription data for these agents were not available. A total of 102 samples (19.2\%) were found positive for illegal drugs, most often amphetamines, cannabinoids, cocaine and morphine. The highest legal drug positive rates were found for analgesics (i.e. naproxen, ibuprofen, tramadol, naloxone) (7.5\%), antidepressants (i.e. mianserin, trazodone) $(4.5 \%)$ and neuroleptics (i.e. clotiapine, pipamperone, prothipendyl) $(4.5 \%)$.

\section{DISCUSSION}

Urine drug tests are a more objective measure of drug use than questionnaires or interviews [9]. In most analytical studies immunoassay techniques such as EMIT $^{\circledR}$ are applied for drug screening, as they are fast and only small sample volumes are required. In these studies only the

Table 3 Comparison of the GC-MS full data to the doctor's prescriptions.

\begin{tabular}{|c|c|c|c|c|c|c|}
\hline \multirow[b]{2}{*}{ Benzodiazepine } & \multicolumn{3}{|l|}{ Prescribed } & \multicolumn{3}{|l|}{ Not prescribed } \\
\hline & No. of patients & Present & Absent & No. of patients & Present & Absent \\
\hline Oxazepam (Seresta ${ }^{\circledR}$ ) & । & । & - & 323 & 51 & 272 \\
\hline Diazepam $\left(\right.$ Valium $^{\circledR}$ ) & 9 & 3 & 6 & 315 & 12 & 303 \\
\hline Temazepam $\left(\right.$ Levanxol $\left.^{\circledR}\right)$ & - & - & - & 324 & 22 & 302 \\
\hline Bromazepam (Lexotan $\left.{ }^{\circledR}\right)$ & 38 & 17 & 21 & 286 & 13 & 273 \\
\hline Alprazolam $\left(\right.$ Xanax $\left.^{\circledR}\right)$ & 14 & 9 & 5 & 310 & || & 299 \\
\hline Lormetazepam $\left(\right.$ Serenase $\left.^{\circledR}\right)$ & 20 & 15 & 5 & 304 & 17 & 287 \\
\hline Flunitrazepam $\left(\right.$ Rohypnol $\left.{ }^{\circledR}\right)$ & I & - & । & 323 & । & 322 \\
\hline Flurazepam (Staurodorm ${ }^{\circledR}$ ) & - & - & - & 324 & 4 & 320 \\
\hline Prazepam $\left(\right.$ Lysanxia $\left.^{\circledR}\right)$ & I & - & I & 323 & 2 & 321 \\
\hline
\end{tabular}

Table 4 Frequency of positive findings for legal and illegal drug classes by GC-MS analysis.

\begin{tabular}{|c|c|c|c|c|c|}
\hline Drug classes & Day 0 & Day 14 & Day 30 & Day 90 & Total \\
\hline No. of samples & 188 & 170 & 135 & 37 & 530 \\
\hline Benzodiazepines & $49(26.1 \%)$ & $64(37.6 \%)$ & 49 (36.3\%) & $12(32.4 \%)$ & $174(32.8 \%)$ \\
\hline Illegal drugs & 45 (23.9\%) & $27(15.9 \%)$ & $26(19.3 \%)$ & $4(10.8 \%)$ & $102(19.2 \%)$ \\
\hline Analgesics & $9(4.8 \%)$ & $16(9.4 \%)$ & $12(8.9 \%)$ & $3(8.1 \%)$ & $40(7.5 \%)$ \\
\hline Antidepressants & $6(3.2 \%)$ & 10 (5.9\%) & 7 (5.2\%) & | (2.7\%) & $24(4.5 \%)$ \\
\hline Neuroleptics & $10(5.3 \%)$ & $5(2.9 \%)$ & $8(5.9 \%)$ & | (2.7\%) & $24(4.5 \%)$ \\
\hline
\end{tabular}


presumptive positive samples are confirmed by specific chromatographic methods $[9,15]$. Depending on the drugs examined and the purpose of the study, immunoassay screening techniques have proved to be very useful as timely and cost-effective results are obtained. However, from Table 1 it is clear that false negative results are often obtained when using immunoassay screening for benzodiazepines compared to GC-MS. For example, 36 positive samples for benzodiazepines (19.1\%) were found on day 0 with EMIT, whereas the true rate is 49 positives (26.1\%), as confirmed by GC-MS. The high false negative rate is the main reason for underestimating the problem of benzodiazepine (mis)use in a prison population when basing conclusions on EMIT results.

An additional drawback of immunoassay is the inability to identify specific benzodiazepines. The GC-MS full data allow not only the compounds with the highest prevalence of (mis)use to be identified as oxazepam and bromazepam, but also allow investigation of whether the frequency of benzodiazepine (mis)use changes over time, based on the number of benzodiazepines taken (Table 2).

The pharmacokinetic profile and metabolism of benzodiazepines are often complex. Oxazepam is a metabolite of several benzodiazepines and also temazepam can be formed during the metabolism of, e.g. diazepam. Both compounds can be used as primary agents as well. The half-lives of benzodiazepines can be up to several days but they vary considerably among individuals. Because of the heavy reliance on hepatic mechanisms the elimination of benzodiazepines may be reduced significantly in patients with inefficient hepatic functioning. Therefore, it should be noted that the presence of oxazepam and temazepam can possibly be attributed to metabolism.

To determine 'illegal' benzodiazepine (mis)use the obtained analytical results need to be compared with the physicians' prescriptions (Table 3). Based on an immunoassay it is impossible to make a correct assessment about the problem of illegally taken benzodiazepines. If the analysis is based on subjects who are not prescribed benzodiazepines, it can only be stated that a subject is using benzodiazepines illegally in the case of a positive test result being obtained. In the subpopulation of prisoners that are on benzodiazepine prescription, no discrimination between prescribed or illegally taken benzodiazepines can be made. Thus studying these two subpopulations can only be conducted effectively with the GC-MS full data. The GC-MS results obtained indicate that illegal benzodiazepine (mis)use is a major problem in the subpopulation that already takes prescribed benzodiazepines $(48.5 \%>>18.6 \%)$.

Another advantage of GC-MS analysis is the possibility of detecting other legal and illegal drugs during the same analysis. The data summarized in Table 4 demonstrate that $23.9 \%$ of the volunteers were positive for illegal drugs on the day of arrival. Although this percentage decreases during detention, illegal drugs remain present and must therefore be circulating in this prison.

\section{CONCLUSIONS}

The present longitudinal study provides valuable information on the (mis)use of benzodiazepines in a Belgian detention house. Performing immunological screening, even followed by GC-MS confirmation of the presumptive positive samples, yields an underestimation of the problem. This is due to the high false negative rate of the immunological screening. GC-MS analysis of all samples is therefore the preferred analytical strategy for the evaluation of benzodiazepine (mis)use. Moreover, additional data on the identity and the number of benzodiazepines are thus obtained. Although it is clear that the work-load and the costs increase significantly, the results obtained give a far more correct view on benzodiazepine (mis)use compared to immunological screening. We suggest that these results should be kept in mind when setting up the analytical strategy for similar longitudinal studies on drug (mis)use or during the interpretation of results obtained, e.g. by immunological screening alone. The authors are convinced that strategies leading to an underestimation of the problem are unhelpful in the combat against drug (mis)use.

\section{ACKNOWLEDGEMENTS}

The authors thank the governor of the detention house for permission to perform this study. The medical staff of the detention house are acknowledged for their efforts to conduct the sampling and G. Vergote for performing the EMIT analyses.

\section{REFERENCES}

1. Korte, T., Pykalainen, J. \& Seppala, T. (1998) Drug abuse of Finnish male prisoners in 1995. Forensic Science International, 97, 171-183.

2. Van Mol, F. (1995) Drugs en gevangenis. In: De Ruyver, B., Vermeulen, G., De Leenheer, A. \& Van Der Straeten Waillet, G., eds. Drugbeleid 2000: Op weg naar een geïntegreerd drugbeleid in België (Drug policy 2000: On the way to an integrated drug policy in Belgium), pp. 305-311. Antwerpen: Maklu Bruylant.

3. Turnbull, G. \& Stimson, V. (1994) Drug use in prison. British Medical Journal, 308, 1716.

4. Morrison, C. L. \& McGee, A. (1996) Access to sterile injecting equipment is important. British Medical Journal, 313, 429.

5. Gore, S. M., Graham, A. \& Ross, A. J. (1996) Prison rights: mandatory drugs tests and performance indicators for prisons. British Medical Journal, 312, 1411-1413. 
6. Mason, D., Birmingham, L. \& Grubin, D. (1997) Substance use in remand prisoners: a consecutive case study. British Medical Journal, 315, 18-21.

7. Power, K. G., Markova, I., Rowlands, A., McKee, K. J., Anslow, P. J. \& Kilfedder, C. (1992) Intravenous drug use and HIV transmission amongst inmates in Scottish prisons. British Journal of Addiction, 87, 35-45.

8. Bird, A. G., Gore, S. M., Hutchinson, S. J., Lewis, S. C., Cameron, S. \& Burns, S. (1997) Harm reduction measures and injecting inside prison versus mandatory drugs testing: results of a cross sectional anonymous questionnaire survey. The European Commission network on HIV infection and hepatitis in prison. British Medical Journal, 315, 21-24.

9. Fraser, A. D., Zamecnik, J., Keravel, J., McGrath, L. \& Wells, J. (2001) Experience with urine drug testing by the Correctional Service of Canada. Forensic Science International, 121, 16-22.

10. Borrey, D., Meyer, E., Lambert, W., Van Calenbergh, S., Van Peteghem, C. \& De Leenheer, A. P. (2001) Sensitive gas chromatographic-mass spectrometric screening of acetylated benzodiazepines. Journal of Chromatography A, 910, 105-118.

11. Borrey, D., Meyer, E., Lambert, W., Van Peteghem, C. \& De Leenheer, A. P. (2001) Simultaneous determination of fifteen low-dosed benzodiazepines in human urine by solidphase extraction and gas chromatography-mass spectrometry. Journal of Chromatography B, 765, 187-197.

12. McNemar, Q. (1947) Note on the sampling error of the difference between correlated proportions or percentages. Psychometrika, 12, 153-157.

13. Wilcoxon, F. (1945) Individual comparisons by ranking methods. Biometrics, 1, 80-83.

14. Mikkelsen, S. L. \& Ash, K. O. (1988) Adulterants causing false negatives in illicit drug testing. Clinical Chemistry, 34, 2333-2336.

15. Skurtveit, S., Abotnes, B. \& Christophersen, A. S. (2002) Drugged drivers in Norway with benzodiazepine detections. Forensic Science International, 125, 75-82. 